\title{
A Survival Case of Super-refractory Status Epilepticus due to Glutamic Acid Decarboxylase Antibodies-associated Limbic Encephalitis
}

\author{
Baoqiong Liu ${ }^{1}$, Yan Zhou ${ }^{2}$, Lingbin Meng ${ }^{1}$, Holly Skinner ${ }^{3}$ \\ 1. Internal Medicine Residency, Florida Hospital, Orlando, USA 2. Critical Care, Medical College In Wisconsin, \\ Milwaukee, USA 3. Neurology, Florida Hospital, Orlando, USA
}

Corresponding author: Baoqiong Liu, lbq0728@gmail.com

\begin{abstract}
Limbic encephalitis (LE) is a neurological syndrome that mainly affects mesial temporal lobes. It may present in association with cancer or infection. Limbic encephalitis associated with glutamic acid decarboxylase antibodies (anti-GAD) is rare. Here, we report a case of anti-GAD limbic encephalitis to heighten the awareness of this rare cause of autoimmune encephalitis. Anti-GAD-associated epilepsy is often poorly responsive to seizure medications. Treatment is challenging. Early initiation of immunotherapy is important.
\end{abstract}

Received 07/31/2018

Review began 08/06/2018 Review ended 08/08/2018 Published 08/10/2018

๑) Copyright 2018

Liu et al. This is an open access article distributed under the terms of the Creative Commons Attribution License CC-BY 3.0., which permits unrestricted use, distribution, and reproduction in any medium, provided the original author and source are credited.
Categories: Internal Medicine, Neurology

Keywords: limbic encephalitis, glutamic acid decarboxylase antibodies, refractory seizures, immunotherapy

\section{Introduction}

Glutamic acid decarboxylase (GAD) is the rate-limiting enzyme in producing gamma-aminobutyric acid (GABA), the main inhibitory neurotransmitter. GAD directed antibody (anti-GAD) is a rare cause of autoimmune limbic encephalitis, with a prevalence of 1.9/100,000. It mainly affects mesial temporal lobes $[1,2]$. Patients usually present with altered mental status, cognitive impairment and refractory seizures [3,4]. We report a case of anti-GAD-associated limbic encephalitis, presenting with super-refractory status epilepticus. Informed consent statement was obtained for this study.

\section{Case Presentation}

A 41-year-old previously healthy Korean man presented with fever and headache for four days, and altered mental status for one day. The family had difficulty waking him up and he was "picking things out of the air". He had no past medical history or any family history of autoimmune diseases. Upon arrival, he was lethargic but without a focal neurologic deficit and had a fever of $100.6^{\circ} \mathrm{F}$. Remarkable labs included white blood cell (WBC) count of $3.55 \times 10^{9} / \mathrm{L}$ with a bandemia of $20 \%$. Routine cerebral spinal fluid (CSF) study results showed elevated WBC count of $72 / \mathrm{ml}$, red blood cell count (RBC) of $24 / \mathrm{ml}$, and protein of $118 \mathrm{mg} / \mathrm{dl}$. CSF glucose was within normal limit at $70 \mathrm{mg} / \mathrm{dl}$. He was empirically treated with vancomycin, ceftriaxone, acyclovir, and dexamethasone. However, his mental status worsened quickly and required intubation.

He was placed on continuous video electroencephalogram (EEG) and found to be in non-convulsive status epilepticus (NCSE) (Figure 1). Extensive infectious workup and cancer screening, including a whole body computed tomography (CT), testicular ultrasound, and flow cytometry of peripheral blood were negative. However, autoimmune workup was remarkable for elevated anti-GAD of $>250 \mathrm{u} / \mathrm{ml}$ and antinuclear antibody (ANA) titer 1:320. Magnetic resonance imaging (MRI) brain demonstrated increased signal in the bilateral mesial temporal lobes (Figure 2). 


\section{Cureus}

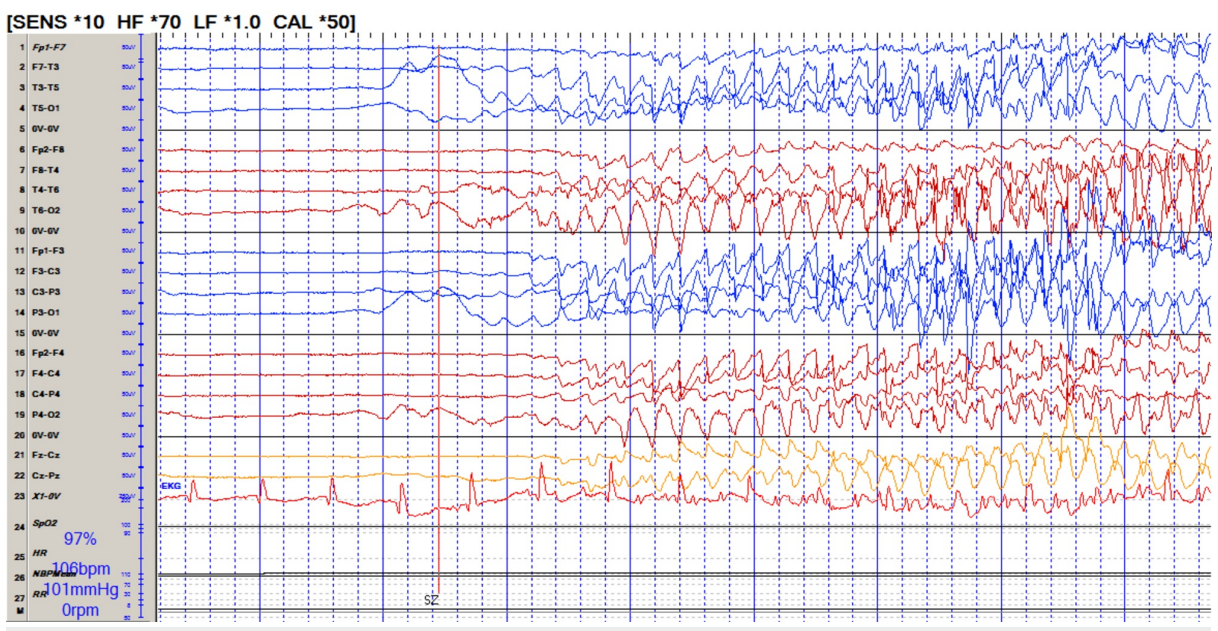

FIGURE 1: Continuous electroencephalogram (EEG) showing breakthrough seizures in spite of pentobarbital-induced burst suppression.

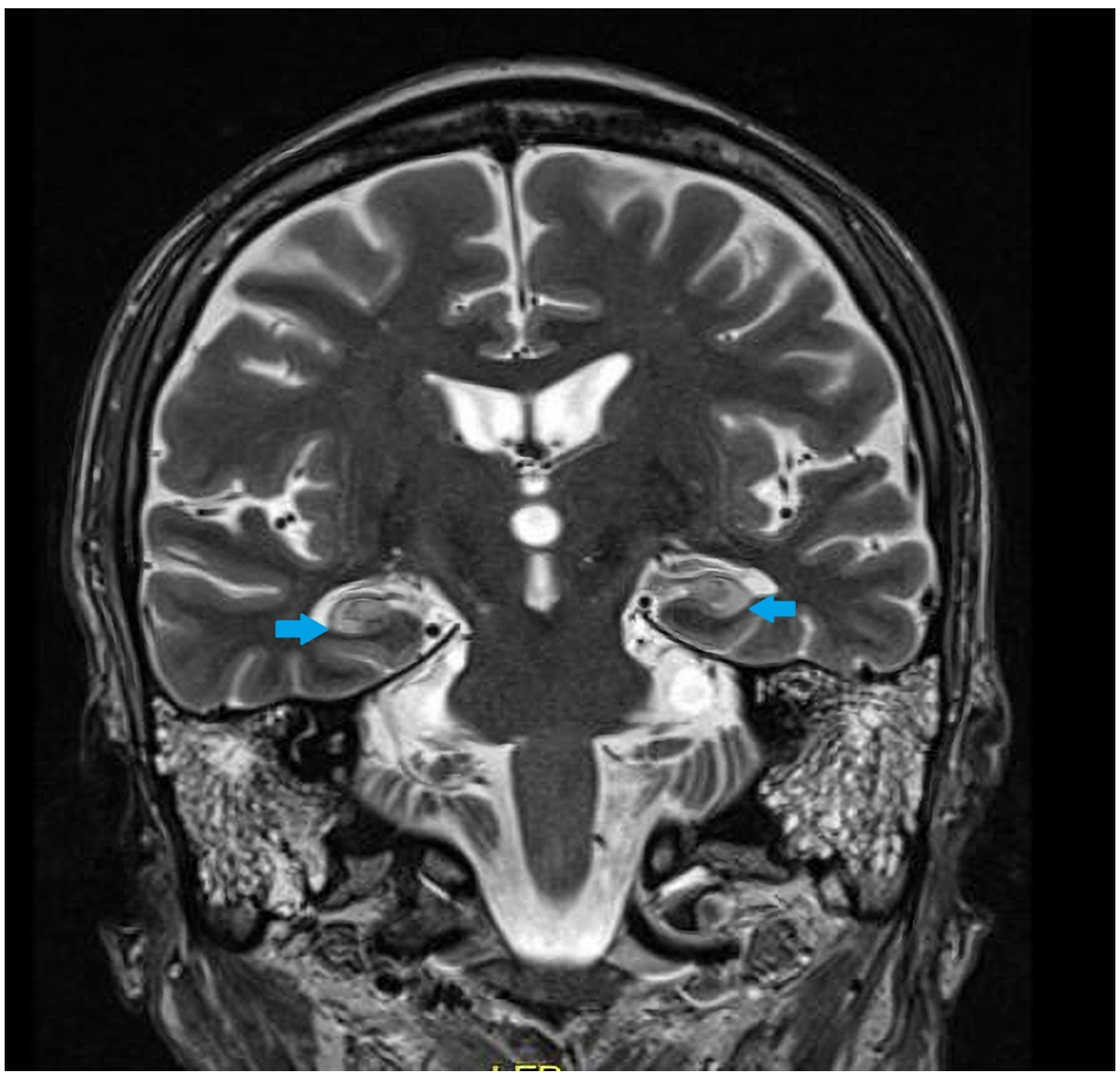

FIGURE 2: Magnetic resonance imaging (MRI) brain coronal T2 image demonstrating increased T2 signal in the bilateral hippocampi (blue arrows).

NCSE continued despite pentobarbital-induced burst suppression necessitating the addition of midazolam and ketamine drips with multiple failed attempts to wean off these sedative-hypnotic medications. All other available intravenous seizure medications (Phenytoin, valproic acid, levetiracetam, phenobarbital and lacosamide) were utilized in various combinations while trying to wean sedative-hypnotic drips. Besides, the patient received a ketogenic diet. 
Diagnosis of anti-GAD-associated autoimmune encephalitis was made based on the clinical course and workup. Immune targeted therapies began with high dose intravenous steroids, then intravenous immunoglobulin (IVIG). Next, he was treated with plasmapheresis which allowed for improvement of seizures activity, tapering of sedative-hypnotic medications and regaining consciousness. However, frequent intermittent seizures continued despite the use of multiple seizure medications. Thus, additional immunotherapies were given. Anakinra (an interleukin 1 receptor antagonist) and Mycophenolic acid were also added.

Three months later, he was decannulated. He improved to be alert and oriented to person and place, with intelligible speech, memory impairment, and mild generalized weakness. Short-term seizure control was achieved using with five seizure medications including oxcarbazepine, phenobarbital, lorazepam, clonazepam, and perampanel. Anti-GAD level was decreased to $17.6 \mathrm{u} / \mathrm{ml}$ at the time of discharge.

\section{Discussion}

GAD antibody has been reported to be in association with both paraneoplastic [5-6] and nonparaneoplastic [7] autoimmune encephalitis. Anti-GAD limbic encephalitis is challenging to diagnose as anti-GAD is not always included in the typical paraneoplastic/autoimmune panels. In patients with antiGAD limbic encephalitis, the CSF anti-GAD antibody titers are often lower than that in the serum. EEG is usually nonspecific. MRI T2-weighted hyperintensity and "swelling" in mesial temporal structure can be found in the acute/subacute phase [6, 7]. For patients with a suspected paraneoplastic syndrome, workup including a whole body CT or a positron emission tomography (PET) scan can be performed to look for tumors. Bone marrow biopsy may also be considered if lymphoma is suspected [8]. In our case, anti-GADassociated autoimmune encephalitis appears to be nonparaneoplastic, as a whole body CT scan, testicular ultrasound, and flow cytometry are negative.

Due to the relative rarity of the disease, there are no prospective trials in this patient population to guide management. All available experience is from case reports. Anti-GAD-associated epilepsy is often poorly responsive to seizure medications [9]. The goal is to reduce immune response and enhance GABAergic activity. Unlike the other autoimmune encephalitis, anti-GAD encephalitis is very resistant to immunotherapy [10]. The non-convulsive status epilepticus of our patient was not well controlled until he received intravenous steroids, IVIG, and subsequent plasmapheresis. Besides, early initiation of immunotherapy should be undertaken before the pathological effects spread to extra-temporal areas which can make the treatment even more challenging.

\section{Conclusions}

Anti-GAD limbic encephalitis is a challenging condition to diagnose and treat. It is not always included in the typical paraneoplastic/autoimmune panels. Anti-GAD-associated epilepsy is often poorly responsive to anti-epileptic drugs. Unlike the other autoimmune encephalitis, anti-GAD encephalitis is relatively resistant to immunotherapy. Immunosuppressants beyond conventional treatments such as intravenous immunoglobulin and plasmapheresis may be necessary and further research is needed to better understand which treatments work best.

\section{Additional Information \\ Disclosures}

Human subjects: Consent was obtained by all participants in this study. Conflicts of interest: In compliance with the ICMJE uniform disclosure form, all authors declare the following: Payment/services info: All authors have declared that no financial support was received from any organization for the submitted work. Financial relationships: All authors have declared that they have no financial relationships at present or within the previous three years with any organizations that might have an interest in the submitted work. Other relationships: All authors have declared that there are no other relationships or activities that could appear to have influenced the submitted work.

\section{References}

1. Wagner J, Schoene-Bake JC, Witt JA, et al.: Distinct white matter integrity in glutamic acid decarboxylase and voltage-gated potassium channel-complex antibody-associated limbic encephalitis. Epilepsia. 2016, 57:475-483. 10.1111/epi.13297

2. Van Ael Y, Amir R, Cras P: Anti-GAD antibodies, a rare cause of limbic encephalitis: a case report . Acta Neurol Belg. 2016, 116:105-107. 10.1007/s13760-015-0493-1

3. Tohid H: Anti-glutamic acid decarboxylase antibody positive neurological syndromes. Neurosciences (Riyadh). 2016, 21:215-222. 10.17712/nsj.2016.3.20150596

4. Sharma A, Dubey D, Sawhney A, Janga K: GAD65 positive autoimmune limbic encephalitis: a case report and review of literature. J Clin Med Res. 2012, 4:424-428. 10.4021/jocmr1080w

5. Boronat A, Sabater L, Saiz A, Dalmau J, Graus F: GABA(B) receptor antibodies in limbic encephalitis and anti-GAD-associated neurologic disorders. Neurology. 2011, 76:795-800. 10.1212/WNL.0b013e31820e7b8d

6. Ariño H, Höftberger R, Gresa-Arribas N, et al.: Paraneoplastic neurological syndromes and glutamic acid decarboxylase antibodies. JAMA Neurol. 2015, 72:874-881. 10.1001/jamaneurol.2015.0749 


\section{Cureus}

7. Matà S, Muscas GC, Naldi I, et al.: Non-paraneoplastic limbic encephalitis associated with anti-glutamic acid decarboxylase antibodies. J Neuroimmunol. 2008, 199:155-159. 10.1016/j.jneuroim.2008.05.015

8. Finelli PF: Autoimmune limbic encephalitis with GAD antibodies . Neurohospitalist. 2011, 1:178-181. $10.1177 / 1941875211413135$

9. Hansen N, Widman G, Witt JA, Wagner J, Becker AJ, Elger CE, Helmstaedter C: Seizure control and cognitive improvement via immunotherapy in late onset epilepsy patients with paraneoplastic versus GAD65 autoantibody-associated limbic encephalitis. Epilepsy Behav. 2016, 65:18-24. 10.1016/j.yebeh.2016.10.016

10. Daif A, Lukas RV, Issa NP, et al.: Antiglutamic acid decarboxylase 65 (GAD65) antibody-associated epilepsy . Epilepsy Behav. 2018, 80:331-336. 10.1016/j.yebeh.2018.01.021 